\title{
Research on the Character Design of Non- Player in Rehabilitation Games
}

\author{
GENG Na,WANG Ying ${ }^{1}$,YU Huan,ZHOU Jiajia \\ Universal Design Institute, School of Art and Design, Zhejiang Sci-tech University
}

\begin{abstract}
The design of reasonable reward mechanisms in exercise games aims to increase people's intrinsic motivation to participate in the games for training so that they can persist for a long time and achieve exercise results. This study developed two game modes based on a prototype of an exercises game for a Kinect interactive device. Two reward mechanisms, in-game rewards and extrinsic rewards, were included in one of the game modes respectively. Twelve older subjects and twelve younger subjects were recruited to test the game. They rated the game using an intrinsic motivation scale. The results showed that young people who played the ingame reward mode scored higher on the motivation scale. In contrast to young people, older people had higher motivation levels when playing the extrinsic reward mode. The results highlight the variability in the effects of in-game and extrinsic rewards on intrinsic motivation across age groups and can help people better design reward mechanisms when developing exercise games.
\end{abstract}

Keywords. Exercise games, rewards mechanisms, intrinsic motivation

\section{Introduction}

The long-term dull and painful treatment process is a great obstacle to patients who have physical dysfunction and need rehabilitation exercise[1]. But since the Internet and video games took off, scientists have taken a cue from people's enjoyment of them and begun to use them in the medical field to attract patients' attention and reduce their perception of pain.

Since the end of the 20th century, scholars have developed many rehabilitation games. Through the analysis of these games, we can find that the existing rehabilitation games only focus on addiction mechanisms, interface design, and reward mechanisms. According to the 2020 China Game Industry Data Report, role-playing games have become the most popular type of games in recent years, and NPC, as a unique design factor, has been increasingly valued. With the increasing number of older players in video games, NPC has become an important tool to help older players adapt, enter, and immerse themselves in the game. However, the NPC design in the game for the elderly is still too rigid, only to play the role of interface guidance and teaching. Therefore, this research takes the NPC design as the research object, through the analysis to extract rules, and applies them in "Hope Farm"—a rehabilitation game. Through the more integral

${ }^{1}$ WANG Ying, Universal Design Institute, School of Art and Design, Zhejiang Sci-tech University, No.928, No.2 Street, Qiantang Distric, Hangzhou,P.R.China; E-mail: winered@zstu.edu.cn. 
NPC characters, the elderly users can form a more healthy and complete relationship, increase emotional experience and fun in the interactive process to prolong their using time, ensure their use frequency, and eventually get a better outcome.

\section{The emergence and development of NPC}

NPC was first created in board games as referees to explain the rules of the game and make decisions, played by neutral players who are not under the control of the players. After the birth of the video game, NPC has also been integrated into it, as an assistant player role. The development of NPC in video games can be divided into three periods: script period, system prompt period, and artificial intelligence period. In the script period, NPC was to accompany the player in the action part of the game, but they would pull away from the game while the player was operating and fighting. So too much of this kind of participation will affect the player's operating experience, and too little will cause a sense of inadequacy. So the NPC design moved towards more integration into the operational process - system prompt period. This period is characterized by the role of partner, by guidance prompting, plot interpretation, conversation, and other ways to intervene in the user's operation process. Examples include Rinker's Goblin in the game The Legend of Zelda and Snake in the game MGS2. As the sense of companionship increases, players also have more requirements for NPC, which pushes development into the artificial intelligence period - The goal is "Infinity to the real thing,".A period that NPC has a clear sense of autonomy, can take on more and more difficult tasks, develop emotions, and even influence players' decisions. The more stereoscopic the NPC is, the stronger the player's sense is of empathy, and the deeper the player's emotional attachment to the game world, which is one of the important reasons why so many people are addicted to video games nowadays[2][3].

NPC is shifting from being things that help players to be more self-aware people. With the popularization of the internet and the progress of science and technology, NPC will be more intelligent and personified. This paper will study the function and type of NPC in games, analyzes the method of using NPC to attract high-frequency users, and applies it to the rehabilitation game to make it have better usability and ease of use, and finally achieve a good rehabilitation effect.

\section{Type and function analysis of NPC}

This research analyzes the NPC in "Genshin" and other popular games to find out how to use NPC to improve the usability and attractiveness of games. "Genshin" is an openworld role-playing game developed in 2020 by miHoYo Technology (Shanghai).In the game, Players will seek relatives through adventures by playing travelers. The game with exquisite character design, novel play, and mysterious vast world settings attract many players. The large NPC system is one of the most important features of the game, playing an important role to help players understand the world, complete the task, experience the plot.

The NPC of "Genshin" can be divided into five types: plot, combat, task, trading, and dialogue. Plot NPC: take on the role of framing the world view and promoting the development of the plot. For example, to introduce the basic situation of the city for 
players, leading the players into the main plot. Combat NPC: take the role of fighting with the players and distributing the rewards. Trading NPC: undertake trading function, provide game material for the player. Dialogue NPC: as the assistant of the plot NPC, carrying on the dialogue with the player. Task-based NPC: assign daily tasks to players.

\subsection{Plot\&Dialogue NPC-Image Building and identity}

The player's first step is usually to build their image, so through which dimension to build a self-image to a large extent affects the player's perception of the game world and the features[4]. As an RPG game," Genshin" first defined the role of the player in the game: not only can control their characters, but also can control NPC,the same goes for Kara and Connor in the game Detroit. Controllable NPC is often the key to the story. By playing these characters, players can get a real sense of what's going on and immerse themselves in the game[5]. While manipulating NPC, players are allowed to view other people's motivations from their views to gain a more objective view and a more mature and complete experience to increase immersion. Therefore, when designing an NPC, we should make it have the same identity, story, and goal setting as the player on the same logic framework, so that it is easier to establish self-image and identity based on the consistency with NPC.

\subsection{Task\&Trading NPC-guidance and assistance}

In" Genshin",the rewards are mainly earned by task NPC(players are rewarded for completing tasks) and by trading NPC (players are rewarded for buying things). Task NPC will give information to guide the players to complete the task. trading NPC sells items through their stores. For example, the current" Genshin" has opened grocery stores in different cities. The goods will reflect the characteristics of the city. Take restaurants as an example, In city A, players can buy western foods like butter and toast, while in City B, they can buy Chinese ingredients like bamboo shoots and lotus seeds.

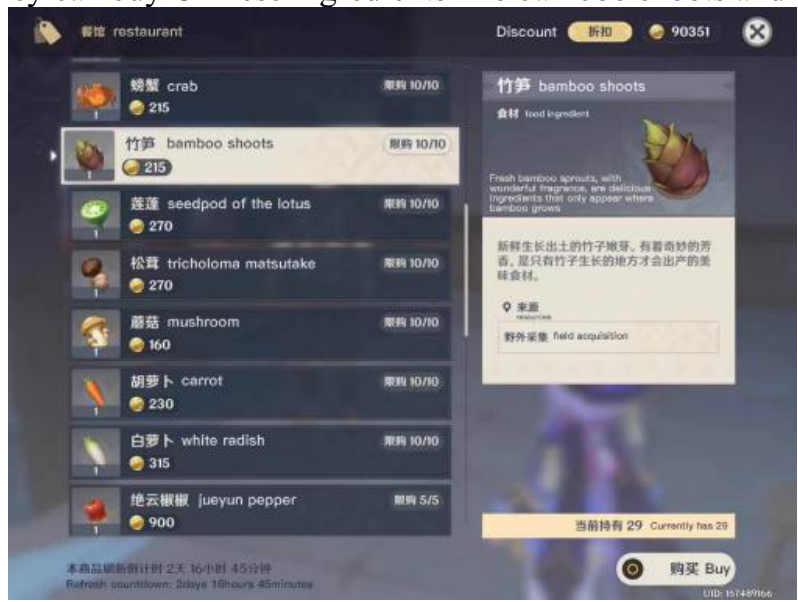

Figure1 : The goods sold in the city shops

The ability to create interest through trading is also shown in original $\sin 2$ : players can find merchants in different locations to trade, and the more successful the transaction, the more favorable the merchant will be to the player, the easier it is for players to buy goods at a good price. On the other hand, if the player repeatedly angered the merchants, 
they would be directly refused to trade with players.

Therefore, the design of such NPC should focus on simplifying the operation process, giving clear operation tips and feedback, and creating real interactive experiences to increase user stickiness.

\subsection{Combat NPC-reward and achievement}

In combat, bright interface colors, explosive motion effects, impassioned music, and dubbing are all used to enhance the user's sensory stimulation. After winning, the largescale rewarding interface will be displayed in front of the players, objects will also be rounded with a highly brilliant light of gold, or purple depending on the level, with which players can get a great sense of satisfaction and achievement.

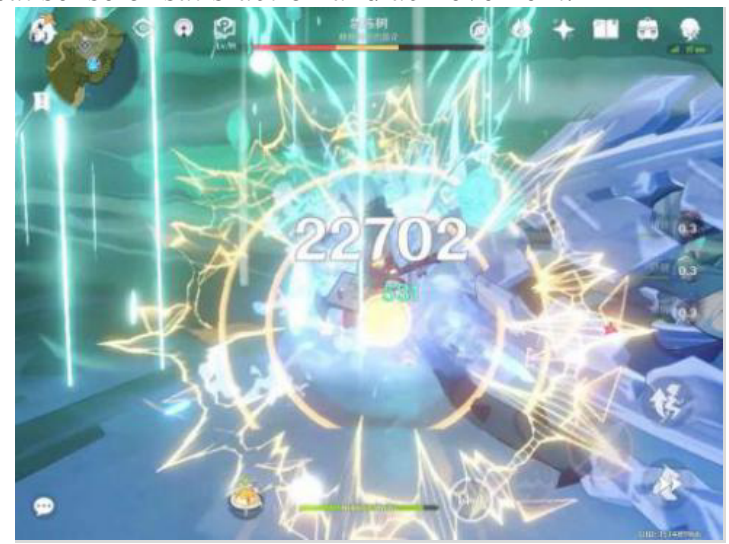

Figure2 : The battle scenes in " Genshin"

However, due to the special physiological mechanism of the elderly, the rehabilitation game needs to be changed: 1 . The visual perception ability of the elderly declines, so the interface with too much brightness and action will cause serious cognitive load and cause discomfort; so it is necessary to properly reduce the proportion of high-brightness color areas, dynamic module, and highlight important information. 2. Because the elderly have fatigability and low ability to withstand pressure, the stimulating confrontation and music should not be too long[6].

\subsection{Summary}

Through the analysis of this article, NPC is divided into five categories: plot, combat, task, trading, dialogue. Plot and dialogue NPC help build self-image perceptions and drive the plot; task and trading NPC provide guidance and assistance to win rewards; combat NPC motivate activity and sense of accomplishment. To sum up, NPC in the rehabilitation game can help players reduce the cost of learning, increase immersion and fun, to maintain user stickiness in the whole cycle, ensure that players have a fixed use frequency, to achieve a good rehabilitation effect.

\section{NPC design in "Hope Farm"}

"Hope Farm" is a kinesthetic interactive game designed by the research team for the hand rehabilitation of the elderly. It exercises by performing various hand 
movements for a certain number of times, duration, and frequency. The game is based on the Leap motion, which uses gesture recognition to translate user hand gestures into a control language for game interaction[7]. For example, the "Open palm-close fist" movement, which trains the neurons in the brain and hemiplegic areas to rebuild[8], is designed to "Confirm" the function, the user needs to perform this action 100 times in the course of completing the game task to complete the training amount once[9]. The game plot is set up for the user to act as a farmer to run a farm. The game can make a rehabilitation plan according to the user's specific condition, set the daily training content and quantity, and record the completion of the user, and through the game, a mechanism to arouse the user's interest, maintain the necessary frequency and length of use, to ensure that the user training on time and in quantity, to achieve good rehabilitation effect.

Through the literature research summary, the old people mainly have the following aspects characteristic psychological:1, the desire for interpersonal communication, especially inter-generational communication between relatives and neighborhood communication. 2. be more sensitive to practical products. 3 . High self-esteem and a desire to achieve self-worth. With the popularity of the internet among the elderly, the aesthetic ability of the elderly is also constantly improving. In Physiology:1, the body function drops, the vision to the color and the dynamic object capture ability becomes bad, the cognition load enhancement. 2. Memory and learning decline.3.A low acceptance of new things and a low willingness to learn.4. Poor coordination.5. physical decline is serious, easy to fatigue.

Therefore, in the design, we need to pay attention to the following points: 1 , pay attention to reflect social, guarantee the player and NPC emotional communication. 2. Give clear, practical rewards and give them promptly. 3, design style can reflect the trend of flu, avoid to the elderly caused by the "Outdated" bad experience. But we should pay attention to the combination of the user's actual life experience, avoid too avant-garde and trendy design style to bring cognitive barriers to it. 4. Avoid secondary messages in the interface and highlight key messages. Use a larger font and a stronger color. However, it should be noted that the font should not affect the aesthetic, color lightness should not be too high, color categories should not exceed three. 5. Add motion effects to key information to capture users attention, but control the number of motion effects and avoid causing visual fatigue and cognitive load.

We designed three types of NPC: Plot\&Task(farm assistant);Task\&Dialogue (neighbors); Trading(merchants).

\subsection{Plot\&Task-farm assistant}

First of all, in the novice guidance phase, the assistant will help the elderly familiar with the game interface, cognitive training gestures. Responsible for explaining the farm world view, operation mode, and other plot information for the user. At the same time, the assistant will also prompt the user to complete the various tasks in the corresponding time. For example, to help players experience the rich social life of the game when a player has no interaction with an NPC after a cumulative 24 hours of use, the farm assistant will actively send out a social invitation to encourage the player to meet new friends. 


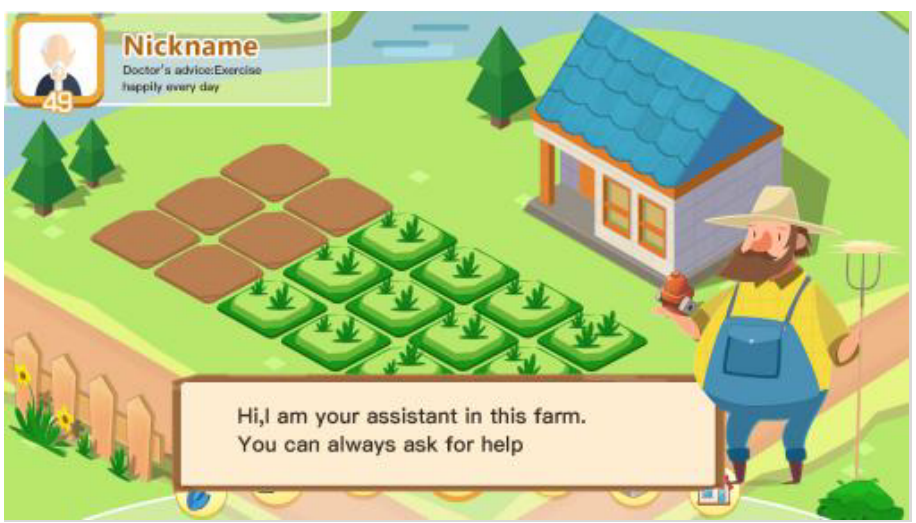

Figure3 : The plans for the farm assistant

\subsection{Task\&Dialogue-neighbors}

The role of dialogue NPC is to communicate in the process of the game to introduce the plot, build complete world settings, mobilize emotional resonance[10]. To avoid mechanizing the task, the farm will match the difficulty of the task with a bigger reward to motivate the player.

Referring to the social habits of the elderly in China, the farm has set up the important role of "Neighbor", which helps users integrate into the virtual society.1.Trust mission. Taking into account the psychological needs of the elderly users, users will receive trust from their neighbors, completing the trust will be rewarded with a thank you letter. 2. Activities. On certain holidays, the game adds activity tasks, allowing players to compete or collaborate with their neighbors to improve neighborhood communication. 3. Surprising encounter. By setting up a sudden plot for the players to simulate interesting small events, forming a common memory between the players and NPC neighbors.

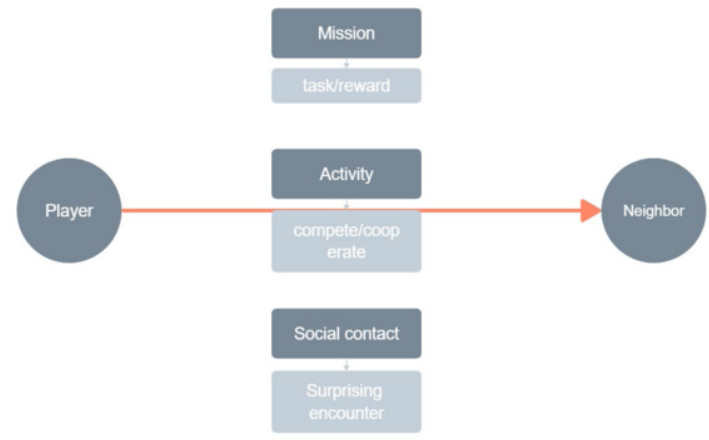

Figure4 : Neighborhood and player diagram

\subsection{Trading-merchants}

Trading NPC offers buying capabilities that mimic the consumption patterns of realworld consumers[11]. Emphasize the concept of "Operation"[12]. Make the game not only a way of entertaining and socializing but also a way for players to value their growth in the game. Players can sell the fruits and vegetables from their farms to merchants for 
coins. In the course of trade, traders can negotiate with merchants to get the highest profit, and there are two main evaluation indexes: personal reputation and vendor favor. In the game, accepting the trust of neighbors and participating in various activities can accumulate the personal reputation of users; the favor of merchants is mainly determined by the number of successful conversations and transactions with merchants, all these will help users to have a good price while trading. The higher the reputation and the favored degree, the merchants and the user's interaction can cause the user's tension and anticipation, thus arouse the user to play the game frequently.

\section{NPC design in "Hope Farm"}

Based on the emergence and development of NPC and the analysis of existing typical game cases, this study divides NPC into five types: plot, combat, task, trading, and dialogue, the role of five types of NPC is analyzed from four aspects: image establishment and identity, guidance and assistance, combat and sense of achievement, plot substitution and emotional connection. The results are applied to the game "Hope Farm". The design of NPC includes four types: plot, task, trading, and dialogue. The farm assistant helps the users to complete the novice guidance and push the plot forward. Neighbors help users build social networks. Merchants are used to forming game management systems, help players form nurturance consciousness, strengthen the dependence of players to game.

Inspired by the actual life of the elderly, this study introduced the unique psychological and physiological characteristics and behavior habits of the elderly into the design of rehabilitation games, the design of NPC in rehabilitation games makes them more willing to use rehabilitation games to complete the amount of training required for rehabilitation. In the future, we will study the virtual social interaction in the rehabilitation game and the emotional identification of the elderly with NPC, to provide some references for the game design.

Acknowledgements This study id supposed by the National College Student Innovation and Entrepreneurship Training Program (Project no.202010338065X)

\section{References}

[1] Chang Kaige, research, and implementation of game design theory for the elderly [d], Lanzhou, Lanzhou University, 2013.

[2] Nikolai O. Veselov、Arthur A. Chubarov、Mikhail N. Roshchin、Lev M. Marder、Stanislav M. Segal、 Alexei V. Samsonovich、Emotional BICA for non-player characters: New empirical data、Procedia Computer Science[J]、Volume 169, 2020. PP 412-422.

[3] Michael Sangyeob Lee、Why are non-player characters not creepy anymore The effect of schema creation on the perception of uncanniness in Oblivion[J]、Journal of Gaming \& Virtual WorldsVolume 10, Issue 1.2018. PP 41-58.

[4] Dong Hui, games, identity and social cohesion: A Study of League of Legends, Shenyang, Shenyang Normal University, 2018.

[5] Analysis of interactive behavior patterns in role-playing games by Chen Qixiang, Yao Di, popular literature and art, 2017,7:267.

[6] Chen Xingbo. Exploration of the elderly user research program for the development of age-appropriate products. Decoration, 2016(10) : 136-137. 
[7] Wang Wei, Pan Rong, discussion on digital humanistic design in product design [J] , Journal of Zhejiang Sci-Tech University, 2006,6(04), 466-469.

[8] Wu Jiajia, Liu Zhen, Xu Huihuang, Liu Tingting, Wang Jin, Chai Yanjie, serious game human computer interaction design for rehabilitation training [J] , Journal of system simulation, 2019,31(5), 909-918.

[9] Wang Ying and Huang Ze. Knowledge Atlas analysis of the development of natural interaction technology [J] . Computer Technology and development, 2021,31(03)

[10] Ricky, social media in games: An Analysis of social communication among mobile gamers, Xi'an, Shaanxi Normal University, 2018.

[11] ZYDA M. From visual simulation to virtual reality to games[J]. Computer,2005,38(9):25-32.

[12] ABT C C. Serious games[M]. New York: University Press of America, 1987:1. 